U. of Iowa $66-25$

\title{
SCATTERING OF ENERGETIC PARTICLES BY LABORATORY PLASMAS *
}

by

David Montgomery and Celso Roqúe ${ }^{* *}$ University of Iowa Iowa City, Iowa

Igor Alexeff Oak Ridge National Laboratory Oak Ridge, Tennessee

\section{Department of Physics and Astronomy University of Iowa Iowa City, Iowa}

$$
\text { June } 1966
$$

\footnotetext{
* This work supported in part by the National Aeronautics and Space Administration under contract NsG-233-62 and in part by the U.S. Atomic Energy Commission. ** Rockefeller Foundation Fellow.
} 


\begin{abstract}
$340^{76}$
Possible experiments on the scattering of energetic particles by laboratory plasmas are discussed. Expressions for scattering coefficients in terms of the electric field autocorrelation function are derived. Sample situations are considered. Whereas incoherent scattering of electromagnetic waves is governed by the charge density auto-correlation function, energetic particle scattering is governed by the electric field autocorrelation function.
\end{abstract}




\section{INTRODUCTION}

Plasma kinetic theories have developed rapidly within the last few years, but have sometimes lacked the focus that would have been provided by a close parallel dcrclopment of experimental tests. Therefore it is of interest to identify in detail classes of experiments which can provide direct tests of various aspects of the theory. The purpose of this note is to point out one such possible measurement for a quantity which is of some current theoretical interest: the spectral density of microscopic electric field fluctuations. (1-7)

Energetic particle beams, which can be sharply defined in both velocity and configuration space, provide a probe for the structure of the microscopic fluctuating electric field in a plasma. For example, the angular spread of a scattered beam provides a direct measure of the integrated spectrum of the electric field auto-correlation function.

The subject under discussion is different from what are now usually called "beam-plasma interactions." The incident beam is assumed to be so tenuous that it is energetically impossible for it to disturb the plasma to any significant extent. Any growth rates for streaming instabilities (which will go to zero as the beam 
density goes to zero) are assumed to be much less than all other frequency scales of interest for the problem. We have essentially a "test-particle" problem. The ideal experiment--not in fact unreasonable with existing electron guns and detectors--would involve scattering one particle at a time.

General expressions for the scattering coefficients are derived in Part II, and various limiting cases are considered in Part III. 


\section{CALCULATION OF THE SCATTERING COEFFICIENTS}

We idealize the plasma as an infinite, spatially-uniform slab, confined between the planes $x=0$ and $x=L$. A monoenergetic beam of charged particles with velocities $\vec{v}_{0}=\vec{v}_{0} \hat{e}_{x}$ strikes the slab at the origin, passes through it, and is detected somewhere in the region $x>L$ (see Fig. 1 ).

The scattered particles have charge-to-mass ratio $\mathrm{q} / \mathrm{m}$, and we either assume $V_{\circ} \gg$ all thermal velocities of the plasma particles of the same species, or else that there are no plasma particles of that species. (This is in order to be sure that the detected particles are the original ones shot in.)

If the number of plasma particles per Debye sphere is high, the occasional large-angle scattering events (i.e., two-body scattering with impact parameters $\lesssim q e / K T)$ can be consistently neglected. The scattering will be largely due to the collective fluctuations in the microscopic electric field and it will be slight. To zeroth order, the incident particles will not be scattered at all; we may calculate the trajectories of the scattered particles as small corrections to the free-flight orbits. 
We first compute formally the scattering of a single incident particle in terms of the exact electric field in the plasma, then introduce statistical averages to bring in the autocorrelation functions. If the electric field in the plasma is $\vec{E}(\vec{x}, t)$, the equation of a particle's motion is

$$
\frac{d^{2} \vec{r}(t)}{d t^{2}}=\frac{g}{m} \quad \vec{E}(\vec{r}(t), t)
$$

(we ignore magnetic forces).

The electric field is most conveniently given as a Fourier integral:

$$
\vec{E}(\vec{x}, t)=\int d \vec{k} d \omega \vec{E}(\vec{k}, \omega) e^{i(\vec{k} \cdot \vec{x}+\omega t)} .
$$

The initial conditions of the orbit may be taken to be $\vec{r}(0)=0, d \vec{r}(0) / d t=\vec{v}_{0}$, and the zeroth approximation to the orbit is $\vec{r}_{0}=\vec{v}_{0} t$.

The first approximation is the solution of

$$
\frac{d^{2} \vec{r}_{1}(t)}{d t^{2}}=\frac{g}{m} \vec{E}\left(\vec{r}_{0}(t), t\right)
$$

which is 


$$
\frac{d \vec{r}_{1}(t)}{d t}=\vec{v}_{0}+\frac{q}{m} \int d \vec{k} d \omega \frac{\vec{E}(\vec{k}, \omega)\left[e^{i\left(\vec{k} \cdot \vec{v}_{0}+\omega\right) t}-I\right]}{i\left(\omega+\vec{k} \cdot \vec{v}_{0}\right)}
$$

and

$$
\begin{aligned}
\vec{r}_{1}(t) & =\vec{v}_{0} t \\
& +\frac{q}{m} \int \frac{d \vec{k} d \omega \vec{E}(k, \omega)}{i\left(\omega+\vec{k} \cdot \vec{v}_{0}\right)}\left\{\frac{\left[e^{i\left(\omega+\vec{k} \cdot \vec{v}_{0}\right) t}-1\right]}{i\left(\omega+\vec{k} \cdot \vec{v}_{0}\right)}-t\right\} .
\end{aligned}
$$

Correct to lowest order, the value of $t$ for which the particle first emerges from the right face of the slab is $L / \mathrm{V}_{0} \equiv t_{0}$, say. Thus the scattering in velocity and position will be

$$
\begin{aligned}
\Delta \vec{v} & \equiv \frac{d \vec{r}_{2}\left(t_{0}\right)}{d t}-\vec{v}_{0} \\
& =\frac{g}{m} \int d \vec{k} d \omega \frac{\vec{E}(\vec{k}, \omega)\left[e^{i\left(\omega+\vec{k} \cdot \vec{v}_{0}\right) t_{0}}-I\right]}{i\left(\omega+\vec{k} \cdot \vec{v}_{0}\right)}
\end{aligned}
$$




$$
\begin{aligned}
\Delta \vec{r} & \equiv \vec{r}_{1}\left(t_{0}\right)-\vec{v}_{0} t_{0} \\
& =\frac{g}{m} \int \frac{d \vec{k} d \omega \vec{E}\left(\vec{k}_{2} \omega\right)}{i\left(\omega+\vec{k} \cdot \vec{v}_{0}\right)} \quad\left\{\frac{e^{i\left(\omega+\vec{k} \cdot \vec{v}_{0}\right) t_{0}}}{i\left(\omega+\vec{k}_{0} \cdot \vec{v}_{0}\right)}-t_{0}\right\} .
\end{aligned}
$$

Formulas (6) and (7) for the displacement in position and velocity of the scattered particle contain much more detail than we can use, because of course $\vec{E}(\vec{k}, \omega)$, the exact electric field, is unknown. However, the experiments will be done on many electrons, so we shall take ensemble averages, and consider expectation values of $\Delta \overrightarrow{\mathrm{v}}$ and $\Delta \overrightarrow{\mathrm{r}}$. We indicate ensemble averages by brackets $(\langle\rangle)$. Since for spatially-uniform plasmas, $\langle\overrightarrow{\mathrm{E}}(\overrightarrow{\mathrm{k}}, \omega)\rangle=0$, the mean values $\langle\Delta \vec{r}\rangle$ and $\langle\Delta \vec{v}\rangle$ vanish. However, the r.m.s. values $\left\langle(\Delta \vec{r})^{2}\right)^{l / 2}$ and $\left((\overrightarrow{\Delta v})^{2}\right)^{1 / 2}$ are non-zero. More general quantities which we can derive from $\Delta \vec{r}$ and $\Delta \vec{v}$, and from which we can derive the mean square deflections, are the tensors 
9

$$
\begin{aligned}
& \langle\Delta \vec{r} \Delta \vec{r}\rangle= \\
& -\frac{\mathrm{q}^{2}}{\mathrm{~m}^{2}} \int \frac{\mathrm{d \vec {k }} d \omega}{\omega+\overrightarrow{\mathrm{k}} \cdot \overrightarrow{\mathrm{V}}_{0}} \int \frac{\mathrm{d \vec {k }} \cdot d \omega^{\prime}}{\omega^{\prime}+\overrightarrow{\mathrm{k}}^{\prime} \cdot \overrightarrow{\mathrm{V}}_{0}}\left\langle\overrightarrow{\mathrm{E}}(\overrightarrow{\mathrm{k}}, \omega) \overrightarrow{\mathrm{E}}\left(\overrightarrow{\mathrm{k}}^{\prime}, \omega^{\prime}\right)\right\rangle \\
& x\left\{\frac{\left[e^{i\left(\omega+\vec{k} \cdot \vec{v}_{0}\right) t_{0}}-I\right]}{i\left(\omega+\vec{k} \cdot \vec{v}_{0}\right)}-t_{0}\right\} \\
& x\left\{\frac{\left[e^{i\left(\omega^{\prime}+\vec{k}^{\prime} \cdot \vec{v}_{0}\right) t_{0}}-1\right]}{i\left(\omega^{\prime}+\vec{k}^{\prime} \cdot \vec{v}_{0}\right)}-t_{0}\right\} \\
& =\frac{\mathrm{q}^{2}}{\mathrm{~m}^{2}} \int \frac{d \overrightarrow{\mathrm{k}} d \omega}{\left(\omega+\overrightarrow{\mathrm{k}} \cdot \overrightarrow{\mathrm{v}}_{0}\right)^{2}}\left|\frac{\left[\mathrm{e}^{i\left(\omega+\overrightarrow{\mathrm{k}} \cdot \overrightarrow{\mathrm{v}}_{\mathrm{o}}\right) t_{0}}-1\right]}{i\left(\omega+\overrightarrow{\mathrm{k}} \cdot \overrightarrow{\mathrm{v}}_{0}\right)}-t_{0}\right|^{2}
\end{aligned}
$$

(8)

and

$$
\begin{aligned}
& \langle\Delta \vec{v} \Delta \vec{v}\rangle= \\
& -\frac{q^{2}}{m^{2}} \int \frac{d \vec{k} d \omega}{\omega+\vec{k} \cdot \vec{v}_{o}} \int \frac{d \vec{k}^{\prime} d \omega^{\prime}}{\omega^{\prime}+\vec{k} \cdot \vec{V}_{0}}\left\langle E(\vec{k}, \omega) E\left(\vec{k}^{\prime}, \omega^{\prime}\right)\right\rangle \\
& x\left\{e^{i\left(\omega+\vec{k} \cdot \vec{v}_{0}\right) t_{0}}\right\} \quad\left\{e^{i\left(\omega^{\prime}+\vec{k}^{\prime} \cdot \vec{v}_{0}\right) t_{0}}\right\} \\
& =\frac{q^{2}}{m^{2}} \int d \vec{k} d \omega\left|\frac{e^{i\left(\omega+\vec{k} \cdot \vec{v}_{0}\right) t_{0}}}{\omega+\vec{k} \cdot v_{0}}\right|^{2}\langle\overrightarrow{\vec{s}}\rangle_{\vec{k} \omega} .
\end{aligned}
$$


In Equations (8) and (9), the tensor quantity $\langle\overrightarrow{\overrightarrow{\mathrm{S}}}\rangle_{\overrightarrow{\mathrm{k}} \omega}$ is the Fourier transform of the autocorrelation tensor $\left\langle\vec{E}(\vec{x}, t) \vec{E}\left(\vec{x}^{\prime}, t^{\prime}\right)\right\rangle$, which obeys the well-known relation

$$
\begin{aligned}
& \left\langle\vec{E}(\vec{k}, \omega) \vec{E}\left(\overrightarrow{k^{\prime}}, \omega^{\prime}\right)\right\rangle \\
& =\langle\overrightarrow{\vec{S}}\rangle_{\vec{k} \omega} \delta\left(\omega+\omega^{\prime}\right) \delta\left(\vec{k}+\vec{k}^{\prime}\right) \\
& =\left\langle\overrightarrow{\mathrm{E}}(\overrightarrow{\mathrm{k}}, \omega) \overrightarrow{\mathrm{E}}^{*}(\overrightarrow{\mathrm{k}}, \omega)\right\rangle \delta\left(\omega+\omega^{\prime}\right) \delta\left(\overrightarrow{\mathrm{k}}+\overrightarrow{\mathrm{k}}^{\prime}\right) .
\end{aligned}
$$

The trace of $8 \pi\langle\overrightarrow{\vec{s}}\rangle_{\vec{k} \omega}$, minus the Fourier-transformed selfenergy of the plasma particles, has the physical interpretation of being the spectral density of the energy in the fluctuating electric field.

Powerful methods have been developed, largely along lines set down by Rostoker, for calculating $\langle\overrightarrow{\vec{s}}\rangle_{\vec{k} \omega}$. It is not the intention here to add to the theory of these quantities, but rather to relate them to concrete observables in the scattering problem. 
III. SCATTERING OVER SEVERAL DEBYE LENGTHS

The extent to which one can go further with expressions (8) and (9) is largely determined by the characteristics of the fluctuation spectimum $\langle\overrightarrow{\vec{s}}\rangle_{\vec{k} \omega}$; These can vary widely.

For situations not greatly different from thermal equilibrium, we expect the contributions to integrals over $\langle\overrightarrow{\vec{s}}\rangle_{\vec{k} \omega}$ to come primarily from the regions

$$
\omega \lesssim|\vec{k}| v_{t h}, \quad\left(|\vec{k}| L_{D}\right)^{-1} \lesssim \text { a few units, }
$$

where $\mathrm{V}_{\text {th }}=$ the maximum thermal velocity in the plasma, and $\mathrm{L}$ $\mathrm{L}_{\mathrm{D}}=\mathrm{k}_{\mathrm{D}}^{-1}=$ the Debye length. If, in addition, the plasma is many Debye lengths thick, and $\vec{k} \cdot \vec{v}_{0} t_{0} \gg 1$ for most of the values of $\vec{k}$, we can approximate (8) and (9) by their asymptotic forms for large $t_{0}$. It is simple algebra to show that for large $t_{0}$,

$$
\langle\Delta \vec{v} \Delta \vec{v}\rangle \cong \frac{2 \pi t_{0} q^{2}}{m^{2}} \int d \vec{k}\langle\overrightarrow{\vec{s}}\rangle_{\vec{k},-\vec{k} \cdot \vec{v}_{0}},
$$

and

$$
\langle\Delta \vec{r} \Delta \vec{r}\rangle \cong \frac{2 \pi}{3} \frac{t_{0}^{3} q^{2}}{m^{2}} \int d \vec{k}\langle\vec{s}\rangle_{\vec{k},-\vec{k} \cdot \vec{v}_{0}} .
$$


The quantity of perhaps most interest is the angular spread produced in the beam. To the order we are going, this is

$$
\left\langle(\Delta \bar{\theta})^{2}\right\rangle=\frac{\left\langle\left(\overrightarrow{\Delta v}_{\perp} \cdot \vec{v}_{\perp}\right)\right\rangle}{V_{0}^{2}}
$$

where $\Delta \vec{v}_{\perp}$ is the component of $\Delta \vec{v}$ perpendicular to $\vec{V}_{0^{\circ}}$. In the present geometry, the full expression is

$$
\left\langle(\Delta \theta)^{2}\right\rangle=2 \pi \frac{q^{2}}{m^{2}} \frac{t_{0}}{V_{0}^{2}} \int d \vec{k}\left\langle\overrightarrow{\vec{s}_{1}}\right\rangle_{\vec{k},-\vec{k} \cdot \vec{v}_{0}},
$$

where

$$
\left\langle\overrightarrow{\vec{s}}_{\perp}\right\rangle_{\vec{k},-\vec{k} \cdot \vec{v}_{0}} \equiv\left\langle s_{y y}\right\rangle_{\vec{k},-\vec{k} \cdot \vec{v}_{0}}+\left\langle s_{z z}\right\rangle_{\vec{k},-\vec{k} \cdot \vec{v}_{0}}
$$

Since $t_{0}=L / V_{0}$, the mean angle of scattering is proportional to the square root of the target thickness, a familiar result from conventional multiple-scattering theory. (8)

A computation of the differential scattering cross section does not appear to be feasible in the present framework. The calculation seems to amount to determining arbitrarily many moments of the probability of scattering into an element of angle d , 
and so far we have only the second moment. The expression for the nth moment involves, for example, the 4n-dimensional Fourier transform of such ensemble averages as

$$
\left\langle\vec{E}(\vec{x}, t) \vec{E}\left(\vec{x}^{\prime}, t^{\prime}\right) \vec{E}\left(\vec{x}^{\prime \prime}, t^{\prime \prime}\right) \ldots n \text { factors }\right\rangle
$$

which rapidly become cumbersome.

A simple application of (13) is recovery of the Spitzer ninety-degree deflection time for a suprathermal particle. For an electron-proton plasma, the theory ${ }^{(1)}$ gives:

$$
\overrightarrow{\langle\vec{s}}\rangle_{\vec{k} \omega}=\frac{2 n_{0} e^{2}}{\pi} \frac{\overrightarrow{k k}}{k^{5}} \frac{\sum_{j} F_{j}(-\omega / k)}{\left|D^{+}(\vec{k}, i \omega)\right|^{2}},
$$

where

$$
\begin{aligned}
& \mathrm{n}_{\mathrm{o}}=\text { number density of electrons and protons } \\
& F_{j}(u)=\int f_{j}(\vec{v}) \delta(u-\vec{k} \cdot \vec{v} / k) d \vec{v} \\
& f_{j}(\vec{v})=\begin{array}{l}
\text { distribution function of } j \text { th species (electron or } \\
\text { proton) }
\end{array} \\
& D^{+}(\vec{k}, i \omega)=1-\lim _{\epsilon \rightarrow 0} \sum_{j} \frac{4 \pi n_{o} e^{2}}{m_{j} k} \int \frac{F_{j}^{\prime}(u) d u}{\omega+k u-i \epsilon} .
\end{aligned}
$$


We may estimate the integral in (13), for the case of equilibrium $f_{j}(\vec{v})$ and $v_{0} \gg$ all thermal velocities, as follows. Most of the contribution will come from the range $\mathrm{k}_{\mathrm{D}} \lesssim|\overrightarrow{\mathrm{k}}| \lesssim \mathrm{k}_{\mathrm{O}}$, where $k_{0}=K T / e^{2}$. For $|\vec{k}|$ much less than $k_{D}$, the $\left|D^{+}\right|^{2}$ gets very large and makes the integrand negligible. Roughly, this part of $\overrightarrow{\mathrm{k}}$-space corresponds to distances greater than a Debye length, which makes a natural limitation on the distance over which the low-frequency part of the electric field can remain correlated. The neglect of the part of $\vec{k}$-space with $|\vec{k}|>k_{0}$ corresponds, as usual, to the neglect of very close encounters. Over nearly all of this range of $\vec{k}$, and for $v_{0}$ large, we have $D^{+}$as $\approx 1$. This gives

$$
\begin{aligned}
\int d \vec{k} & \left\{\left\langle\overrightarrow{\vec{s}_{\perp}}\right\rangle \vec{k},-\vec{k} \cdot \vec{v}_{0}\right\} \\
& \approx \frac{2 n_{0} e^{2}}{\pi} \quad(2 \pi) \int_{k_{D}}^{k_{0}} k^{2} d k \int_{-I}^{+1} d(\cos \theta) \frac{\sin ^{2} \theta}{k^{3}} \Sigma_{j} F_{j}\left(-V_{0} \cos \theta\right) \\
& =\frac{4 n_{0} e^{2}}{V_{0}}\left(\ln \frac{k_{0}}{k_{D}}\right) \int_{-V_{0}}^{V_{0}}\left(I-\frac{\xi^{2}}{V_{0}^{2}}\right) \Sigma_{j} F_{j}(-\xi) \\
& \approx \frac{8 n_{0} e^{2}}{V_{0}} \ln \left(\frac{k_{0}}{k_{D}}\right),
\end{aligned}
$$


so that

$$
\left\langle(\Delta \theta)^{2}\right\rangle \approx 16 \pi \frac{q^{2} e^{2}}{m^{2}} \frac{t_{0}}{V_{0}^{3}} \quad n_{0} \ln \left(\frac{k_{0}}{k_{D}}\right) .
$$

Setting $\left((\Delta \theta)^{2}\right)=(\pi / 2)^{2}$, and sulving for to gives

$$
t]_{90^{\circ} \text {-scattering }}=\frac{\pi}{64} \frac{\mathrm{m}^{2} \mathrm{v}_{0}^{3}}{\mathrm{n}_{0} \mathrm{e}^{2} \mathrm{q}^{2} \ln \left(\mathrm{k}_{\mathrm{o}} / \mathrm{k}_{\mathrm{D}}\right)} .
$$

This agrees, up to a numerical factor of order unity, with the Spitzer ninety-degree deflection time for the same situation. (6) From (15), we may also inquire into the thickness of a plasma necessary to scatter a beam through an angle $\varphi$, say:

$$
L=\frac{1}{4 \pi} \frac{T^{2} \varphi^{2}}{n_{0} q^{2} e^{2} \ln \left(k_{0} / k_{D}\right)}
$$

where $\mathrm{T}=\mathrm{mV}_{0}^{2} / 2$ is the particle kinetic energy.

Formula (17) illustrates what may prove to be one of the more annoying difficulties in the experiment: when $\mathbb{T}$ is large enough for the scattered particles to be easily countable, and $\varphi$ is measurably large, (17) only leads to reasonable lengths when $\mathrm{n}_{\mathrm{O}}$ is very large. 
For example, if we estimate $\ln \left(k_{0} / k_{D}\right)$ at about 10 , require $\varphi$ to be 2 degrees, and let $\mathrm{n}_{0} \approx 10^{14}$, then $\mathrm{L} \approx 5 \times 10^{4} \mathrm{~cm}$ for scattering a kilovolt electron--a prohibitively large plasma. Bringing the energy down to $50 \mathrm{eV}$ gives $\mathrm{L} \approx 10 \mathrm{~cm}$, $\mathrm{vut} 50 \mathrm{eV}$ is getting close enough to the thermal energy of most realizable plasmas that problems might well arise in distinguishing the scattered particles from the plasma particles.

Perhaps the best solution to the dilemma is to scatter only positive ions of species which are not present in the plasma. The energies of these could be made low enough to reduce $\mathrm{L}$ to reasonable values without risk of confusion with the plasma particles. If the ions were multiply charged, L would of course be further decreased, due to the $\mathrm{q}^{2}$ in the denominator of (17).

Some circumstances can act to greatly enhance plasma fluctuations and thus greatly increase $\left\langle(\Delta \theta)^{2}\right\rangle$. Most notably, as an unstable situation is approached, $\left|D^{+}\right|^{-2}$ develops a resonance which dominates the integrals over $\left\langle\overrightarrow{\overrightarrow{\mathrm{S}}} \overrightarrow{\vec{k}}_{\overrightarrow{\mathrm{k}}},-\overrightarrow{\mathrm{k}} \cdot \overrightarrow{\mathrm{V}}_{\mathrm{O}}\right.$, eventually diverging as the stability boundary is reached. (A plasma carrying a current slightly less than that required for the onset of instability is the most obvious example.) The greatly enhanced scattering of the test beam can act as a plasma probe to signal the approach of an instability, and may prove to be the most important application of the energetic particle-scattering technique. 


\section{ACKNOWLEDGEMENTS}

This work was supported in part by the National Aeronautics and Space Administration grant NsG-233-62, and in part by the U.S. Atumic Anergy Commission. 


\section{REFERENCES}

(1) N. Rostoker, Nucl. Fusion 1 , 101 (1960).

M. N. Rosenbluth and N. Rostoker, Phys. Fluids 2, 776 (1962)

(2) J. P. Daugherty and D. T. Farley, Proc. Roy Soc. (London) A259, 79 (1960).

E. Saltpeter, Phys. Rev. 120, 1528 (1960).

J. A. Fejer, Can. J. Phys. 38, 1114 (1960).

(3) K. L. Bowles, J. Res. Natl. Bur. Std. 66D, 395 (1962).

(4) J. Weinstock, Phys. Rev. 139, A388 (1965)

J. Weinstock, Phys. Fluids 2, 621 (1966).

(5) W. B. Thompson, "An Introduction to Plasma Physics," Pergamon Press, New York, and Addison-Wesley Publishing Co., Reading, Mass., 1962.

(6) D. Montgomery and D. Tidman, "Plasma Kinetic Theory," McGraw-Hill Publishing Co., New York, 1964. The auto-correlation functions used in this paper are obtained from those in this book by dividing by $(2 \pi)^{4}$. 
(7) A. Eviatar, University of Maryland Tech. Note BN-429

$$
\text { (January, 1966) (to be published), and }
$$

P. A. Sturrock, Phys. Rev. 141, 186 (1966).

(8) E. Fermi, Nhaclear Physics, Iniversity of Chicago Press, Chicago, Illinois, 1950. 


\section{FIGURE CAPTION}

Figure 1. Geometry of the scattering. The incident beam strikes the plasma from the left at the origin. 
G 66-400

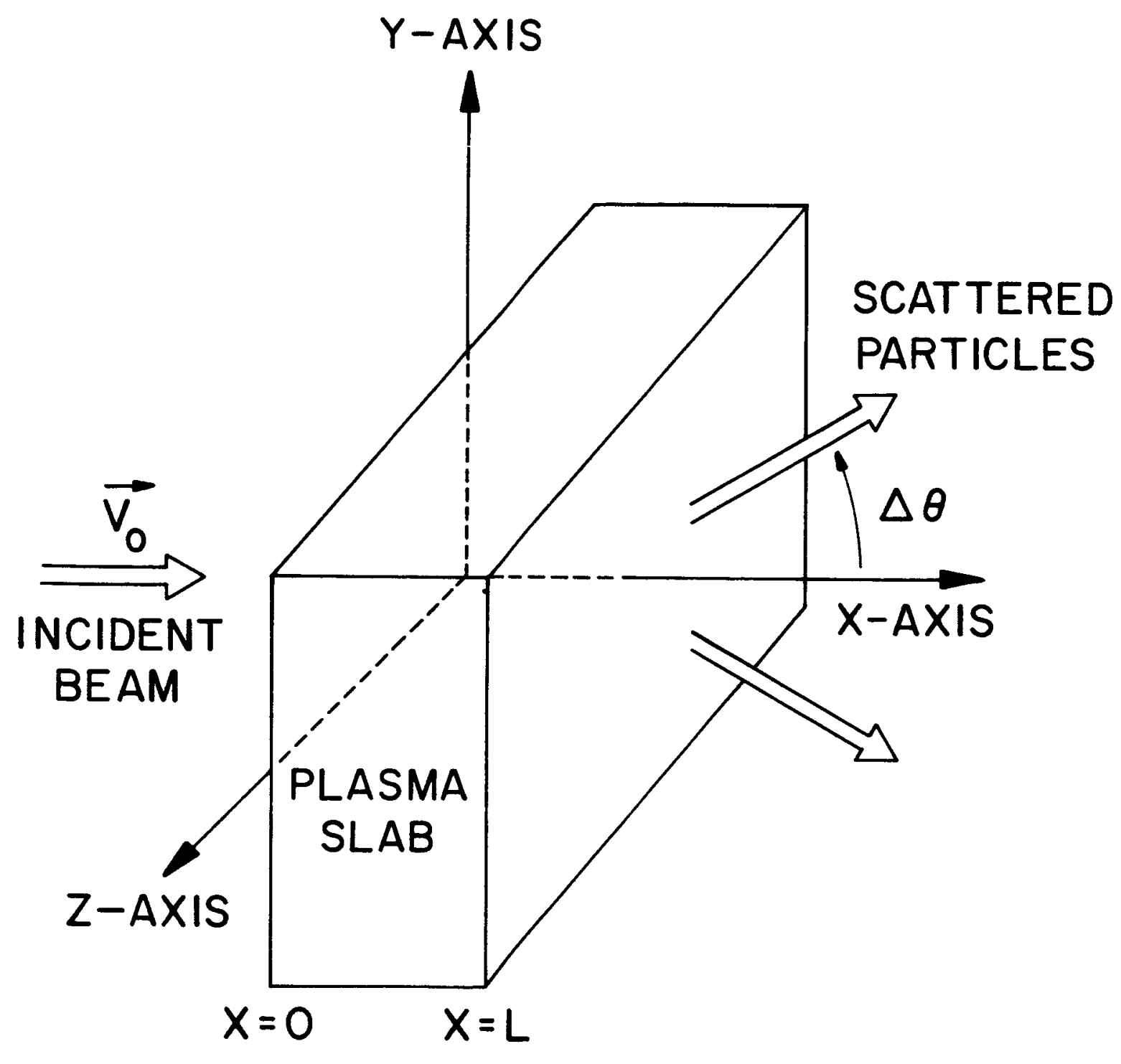

FIGURE I 